\title{
Improving Measures of Hip Fracture Wait Times: A Focus on Ontario
}

Jennifer Frood and Tracy Johnson

\begin{abstract}
In 2009-2010, a "time of surgery" data element was added to CIHI's Discharge Abstract Database enabling a more precise calculation of patient wait times for hip fracture repair, measured in hours rather than days. Using an Ontario sample, we explored this more precise calculation for the first three quarters of 2009-2010 (April to December), and the impact of adding wait times in the emergency department (ED) to the total wait. When we linked emergency department and in-patient care wait times, the percent of patients meeting the benchmark of $\mathbf{4 8}$ hours dropped from $\mathbf{7 8 \%}$ (when the start time was admission to an acute care bed) to $71 \%$.
\end{abstract}

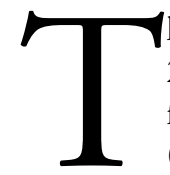

housands of Canadians break a hip each year. In 2005-2006, over 25,000 surgeries to repair hip fractures were performed in Canadian hospitals (CIHI 2007). Hip fractures pose significant health risks for patients, particularly seniors. About $7 \%$ of seniors admitted with a hip fracture died in hospital within 30 days of admission (CIHI 2007). Research suggests that patients, in general, benefit from timely hip repair surgery (Moran et al. 2005). Timely repairs are associated with reduced morbidity, mortality, pain and length of stay in hospital, as well as improved rehabilitation (Orosz et al. 2004). Recognizing the benefit of timeliness, in December 2005 provincial health ministers of Canada committed to a benchmark of surgical repair of hip fractures within 48 hours (Ontario Ministry of Health and Long-Term Care 2005).

Initially, there were practical challenges in measuring progress towards this benchmark. Most data collection systems were designed to capture "date of surgery" only. This was effective for most of the five wait time priority area procedures, where waits were measured in days, weeks or months. However, for emergency procedures like hip fracture repair, measurement in hours was required so data sets need to include a "time" of repair as well as date. In 2009-2010, the collection of "time of surgery" was added to the CIHI discharge abstract submitted to CIHI, which allows for a more precise calculation of patient waits for hip fracture repair.

There are many places to measure wait times along the continuum. When your wait time starts depends a great deal on your perspective. From the patients' perspective, the wait starts when they fall. Wait times start from a data collection perspective when the patient enters the hospital system. There is also variability within the data collection perspective as there are a number of dates and times along the patient continuum of care that allow us to monitor patient flow through the system (Figure 1).

Currently, CIHI holds pan-Canadian data for in-patient and day surgeries. Previous estimates of wait times have focused
Figure 1. Wait times for care or decisions from emergency department to surgery

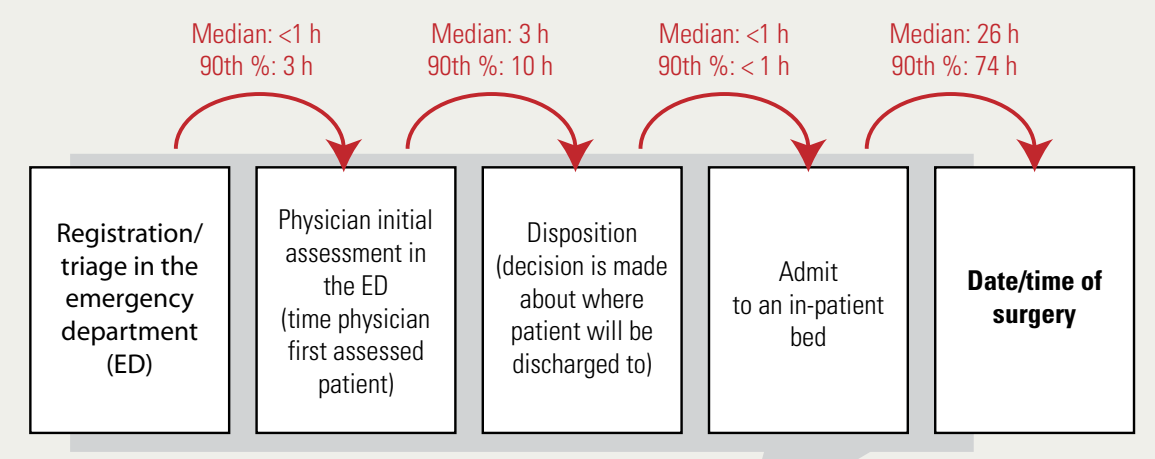

Sources: Canadian Institute for Health Information, Discharge Abstract Database (DAD), 2009-2010, and National Ambulatory Care Reporting System (NACRS), 2009-2010. 
mainly on in-patient care as these data are available nationally; however, CIHI has a growing body of emergency department data. By adding information about wait times in EDs, we can better understand the various wait times across the continuum such that bottlenecks/problem areas can be addressed. In this study, we only look at Ontario, where complete information is available on emergency cases, to see how rolling back the start time on the wait for hip fracture surgery affects achievement of the benchmark of 48 hours.

\section{From the patients" perspective, the wait starts when they fall.}

\section{Data Sources and Methods}

Our study patients were adults who were admitted to an in-patient bed in an acute care facility with a pre-admission diagnosis of hip fracture and who had a surgical hip repair (see Table 1). Patients without a health card number or those who experienced a hip fracture while in hospital were excluded. In-patient data were obtained from CIHI's Discharge Abstract Database (DAD) which contains demographic, administrative and clinical data for all in-patient acute care hospital discharges across Canada, excluding Quebec. Patients discharged between April 1 and December 31,2009 , with a hip fracture and surgical repair of the fracture from an Ontario acute care facility were identified and included in the study.

These in-patients were then linked to their emergency department data in order to track patients from registration in the ED to hip fracture repair surgery in an acute care facility. As patients may be transferred between facilities over the course of care, all transfers were linked from first contact to related surgical repair, that is, transfers were treated as a single admission. Emergency department information was obtained from CIHI's National Ambulatory Care Reporting System (NACRS). Patients discharged between April 1 and December 31, 2009, from an Ontario emergency care facility were included in this study.

As the time of surgery is a new data element and a full data collection cycle has not been completed, this study used "open year data" - meaning the dataset represents less than one full year of data and will not have been subject to the complete cycle of quality validation. At the time of analysis it was estimated that CIHI had received $99 \%$ of acute care abstracts and $101 \%$
Table 1. Definitions for inclusion

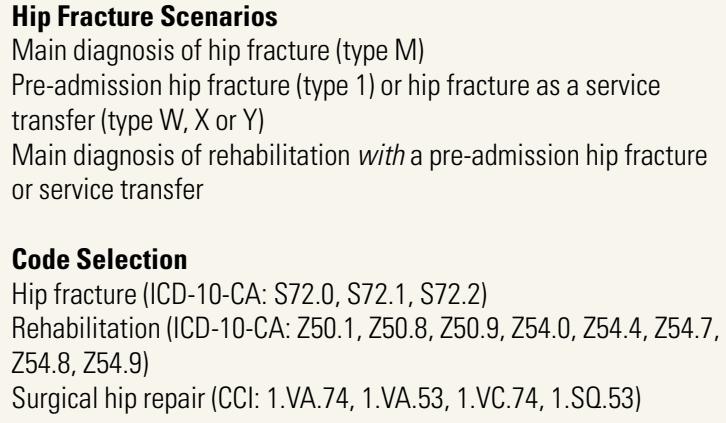

\section{Code Selection}

Hip fracture (ICD-10-CA: S72.0, S72.1, S72.2)

Rehabilitation (ICD-10-CA: Z50.1, Z50.8, Z50.9, Z54.0, Z54.4, Z54.7,

Z54.8, Z54.9)

Surgical hip repair (CCI: 1.VA.74, 1.VA.53, 1.VC.74, 1.S0.53)

$\mathrm{CCl}=$ Canadian Classification of Health Interventions; ICD-10-CA = International

Classification of Diseases, 10th Revision, Canadian Enhancement.
Table 2. Wait times and percentage meeting benchmark for different start times

\begin{tabular}{|l|c|c|c|}
\hline Data by Wait Segment ( $\mathbf{n = 6 , 6 1 7 )}$ & $\begin{array}{c}\text { Median Wait } \\
\text { Time (h) }\end{array}$ & $\begin{array}{c}\text { 90th } \\
\text { Percentile (h) }\end{array}$ & $\begin{array}{c}\text { \% Meeting 48 h } \\
\text { Benchmark }\end{array}$ \\
\hline $\begin{array}{l}\text { Wait from hospital admission (index) to } \\
\text { hip fracture surgery }\end{array}$ & 26 & 74 & 78 \\
\hline $\begin{array}{l}\text { Wait from ED registration to hip fracture } \\
\text { surgery }\end{array}$ & 32 & 81 & 71 \\
\hline
\end{tabular}

Sources: Canadian Institute for Health Information, Discharge Abstract Database (DAD), 2009-2010, and National Ambulatory Care Reporting System (NACRS), 2009-2010. (i.e., more visits in 2009 than 2008) of ED abstracts, relative to the same nine months in the previous year in Ontario. There were very few $(<1 \%)$ missing or invalid times submitted in the "time of surgery" field.

There were 7,258 patients hospitalized in Ontario with hip fractures and repairs in the first nine months of 2009-2010. Most patients, 6,617 or $91 \%$ of the patients, were admitted through and received some treatment in emergency departments. The remaining were excluded from this study because (1) they were admitted directly into an in-patient bed bypassing the $\mathrm{ED}(3 \%)$ or (2) the record indicated the patient was admitted to in-patient care from the ED but there was no corresponding ED record (6\%).

\section{Key Findings}

There were 6,617 patients admitted to an Ontario emergency department and hospitalized for a hip fracture repair in the first nine months of 2009-2010. 
Figure 2. Total timeline of waits for hip fracture surgery

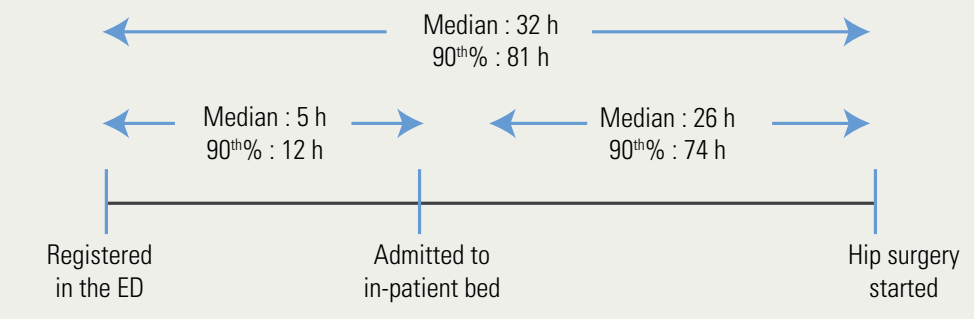

Sources: Canadian Institute for Health Information, Discharge Abstract Database (DAD), 2009-2010, and National Ambulatory Care Reporting System (NACRS), 2009-2010.

\section{Summary}

While the majority of time spent waiting for hip repair occurs while the patient is in an in-patient bed, the addition of time spent waiting in the ED does have an impact on the percent of patients receiving surgery within the prescribed 48 hours $(71 \%$ versus $78 \%)$. This study demonstrates that the addition of "time of surgery" allows for a more accurate assessment of whether hip fracture patients are receiving care within the nationally established benchmark of 48 hours. Using time of surgery and measuring the wait from two different start times (admission to an in-patient bed or admission to the ED) provides more precise measurement of patient waits across the continuum. The addition of

The benchmark of hip fracture surgery in 48 hours is often applied to the in-patient wait only since these data are available across the country. Looking at the in-patient portion of the wait, $78 \%$ of hip fracture repairs in Ontario were completed within the benchmark of 48 hours (Table 2). Adding the time waiting in $\mathrm{ED}$ decreases this percentage to $71 \%$ of patients receiving surgery within the prescribed benchmark. The median wait in Ontario was six hours longer when the time spent waiting in ED was included (26 hours vs. 32 hours).

Looking at the overall timeline, 50\% (the median) of all patients waited 32 hours or less from admission to ED to surgery (Figure 2). This overall wait is composed of transfer time from one hospital to another, time spent waiting in the $\mathrm{ED}$, as well as time in an in-patient bed. Patients spend the majority of their time waiting in an in-patient bed (26 hours) but half of the patients spent five hours or more waiting in the ED. A small proportion of patients (10\%) waited longer than 12 hours in ED and more than 74 hours in a bed before their surgery. Half of patients spend $15 \%$ of their wait for hip fracture surgery in the ED.

There is a large difference between the median (50th percentile) and the 90th percentile waits and this difference remains whether the measure includes or excludes waits in the ED. Patients who require treatment prior to surgery to stabilize medical conditions are more likely to wait longer than others for their repair (Charalambous et al. 2003). Other factors influencing longer waits include at least one transfer, admission to larger, high-volume hospitals, time of admission (e.g., afternoon and evening), as well as day of week of admission (CIHI 2007).

Once it is decided that a patient needs to be admitted for surgery, very little time is spent waiting in $\mathrm{ED}$ for an in-patient bed (see Figure 1). Only $10 \%$ of patients waited longer than 30 minutes for a bed, and 5\% waited longer than one hour. The majority of time waiting in ED was prior to the decision to admit to an in-patient bed, presumably waiting for tests such as radiography confirming the presence of a hip fracture and the need for surgery. "time of surgery" will enable tracking of waits for other types of emergency surgery. This, in combination with the use of "open year" data, can provide more timely and accurate information, which is important for system improvement. HQ

\section{References}

Canadian Institute for Health Information. 2007. Health Indicators, 2007. Ottawa, ON: Author. Retrieved May 5, 2010. <http://secure. cihi.ca/cihiweb/dispPage.jsp?cw_page=download_form_e\&cw_ sku=07HLTHINDPDF\&cw_ctt=1\&cw_dform=N>.

Charalambous, C.P., S. Yarwood, C. Paschalides, I. Siddique, P. Hirst and A. Paul. 2003. "Factors Delaying Surgical Treatment of Hip Fractures in Elderly Patients." Annals of the Royal College of Surgeons of England 85(2): 117-19.

Moran, C.G., R.T. Wenn, M. Sikand and A.M. Taylor. 2005. "Early Mortality after Hip Fracture: Is Delay before Surgery Important?" Journal of Bone and Joint Surgery, American 87: 483-89.

Ontario Ministry of Health and Long-Term Care. 2005. First Ever Common Benchmarks Will Allow Canadians to Measure Progress in Reducing Wait Times. Toronto, ON: Author. Retrieved May 5, 2010. $<$ www.health.gov.on.ca/english/media/news_releases/archives/nr_05/ nr_121205.html>.

Orosz, G.M., J. Magaziner, E.L. Hannan, R.S. Morrison, K. Koval and M. Gilbert. 2004. "Association of Timing of Surgery for Hip Fracture and Patient Outcomes." Journal of the American Medical Association 291(14): 1738-43.

\section{About the Authors \\ Jennifer Frood, MSc, is a project lead with the Canadian Institute for Health Information Emerging Issues team. Jennifer is responsible for performing the analyses and provides methodological support to projects.}

Tracy Johnson, BScPT, MBA, is manager of the Canadian Institute for Health Information Emerging Issues team. 


\section{Longwoods.com}

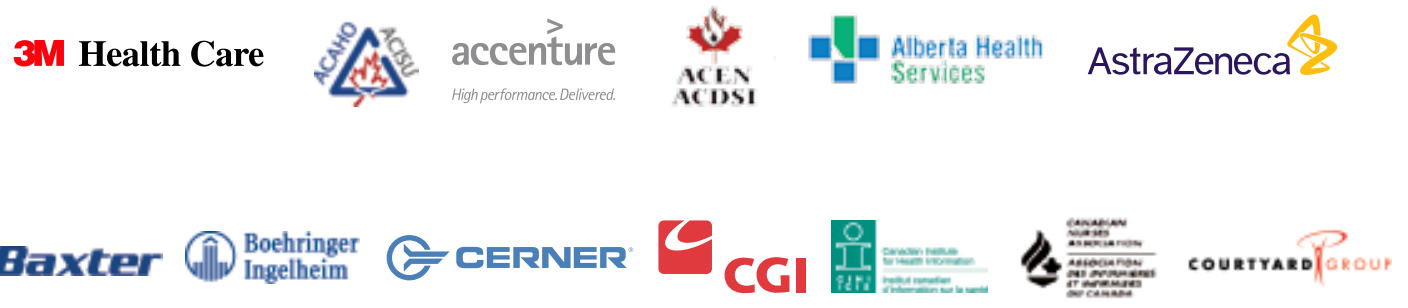

E COVIDIEN Fe Healthcare

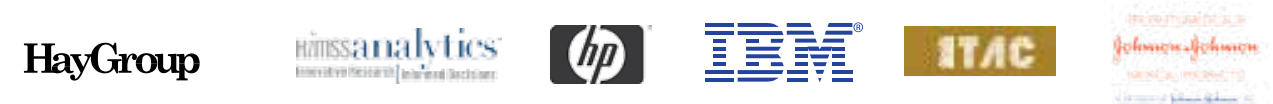

Repres

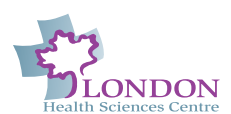

MCKESSON

Ganada

Microsoft ":":": ODGERS BERNDTSON

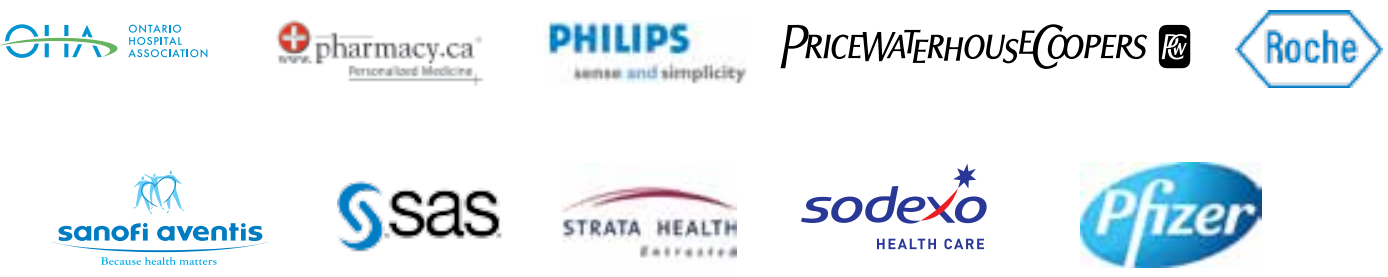
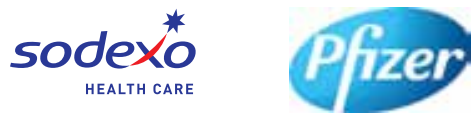

Q $\begin{aligned} & \text { Health Policy, Manayement \& Evaluarion } \\ & \text { UNIVERSITY OF TORONTO }\end{aligned}$

$$
\text { health authority }
$$

\section{Rotman}

University of Alberta

UBC
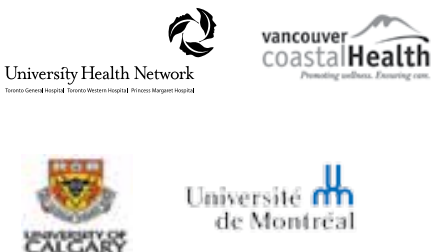

Longwoods journals are published in partnership with our readers, our editors, our advisory boards, our authors as well as healthcare organizations and their suppliers of solutions and services. We value this participation in and dedication to leadership and knowledge. They enable us to present new ideas, policies and best practices essential to healthcare management, practice, education, research and innovation. It is a measure of their support for learning. Nothing can be more fundamental to the progress of healthcare. 